(C) 2019 IEEE

20th International Symposium on Power Electronics - Ee 2019

\title{
Comparison of two Modular Multilevel Converter Internal Energy Balancing Methods
}

M. Basic., S. Milovanovic, and D. Dujic

This material is posted here with permission of the IEEE. Such permission of the IEEE does not in any way imply IEEE endorsement of any of EPFL's products or services. Internal or personal use of this material is permitted. However, permission to reprint / republish this material for advertising or promotional purposes or for creating new collective works for resale or redistribution must be obtained from the IEEE by writing to pubs-permissions@ieee. org. By choosing to view this document, you agree to all provisions of the copyright laws protecting it. 


\title{
Comparison of two Modular Multilevel Converter Internal Energy Balancing Methods
}

\author{
Miodrag Basić, Stefan Milovanović and Dražen Dujić \\ Power Electronics Laboratory - PEL \\ École Polytechnique Fédérale de Lausanne - EPFL \\ Station 11, CH-1015 Lausanne \\ miodrag.basic@epfl.ch,stefan.milovanovic@epfl.ch,drazen.dujic@epfl.ch
}

\begin{abstract}
Although challenging, control of the modular multilevel converter internal variables has been addressed using several different approaches so far. With the aim of maintaining the submodules' energies within a predefined band, the socalled circulating current control must be properly established. However, the performance of the converter energy balancing controllers depends on the way circulating current references are generated. Energy balance of the modular multilevel converter implies two directions equilibrium. Namely, horizontal and vertical balancing can be achieved by means of different circulating current components, which allows for the decoupled control of the two. This paper presents a thorough comparison of the two methods guaranteeing balancing of the converter energies without causing any DC link current distortions. To validate the analyses presented within the paper, results acquired by conducting a detailed set of simulations are presented.
\end{abstract}

\section{INTRODUCTION}

In the High Voltage (HV) and Medium Voltage (MV) domain, once an application power level reaches the order of $100 \mathrm{MW}$, technology limitations of monolithic converter structures emerge [1]. The voltage level of commercially available semiconductor devices nowadays reaches as high as $6.5 \mathrm{kV}$ consequently affecting the scalability of converter topologies. For instance, Neutral Point Clamped (NPC) and Cascaded H-Bridge (CHB) converters require either topology or input transformer modifications to increase the number of voltage levels, respectively. Therefore, these topologies can not be considered truly modular. Modular Multilevel Converter (MMC), on the other hand, was firstly introduced in [2] as an inherently scalable, fully modular structure. Nowadays, the topology is widely deployed in scenarios where two AC systems are to be interconnected, e.g. point-to-point High Voltage Direct Current (HVDC) bulk energy transmission systems [3], Static Synchronous Compensators (STATCOMs) [4], Static Frequency Converters (SFCs) for railway interties [5], etc. Perspective for future use is also seen in high-power four-quadrant drives for Pumped Hydro Storage Plant (PHSP) applications [6], [7].

The basic building block of an MMC is the Submodule (SM), predominantly either in Full-Bridge (FB) or Half-Bridge (HB) configuration, as presented in Fig. 1. Converter branches are realized as clusters of $N_{\mathrm{SM}}$ series connected two-terminal SMs, and branch inductances $L_{\mathrm{br}}$ having the dual purpose (protection along with the control of in-

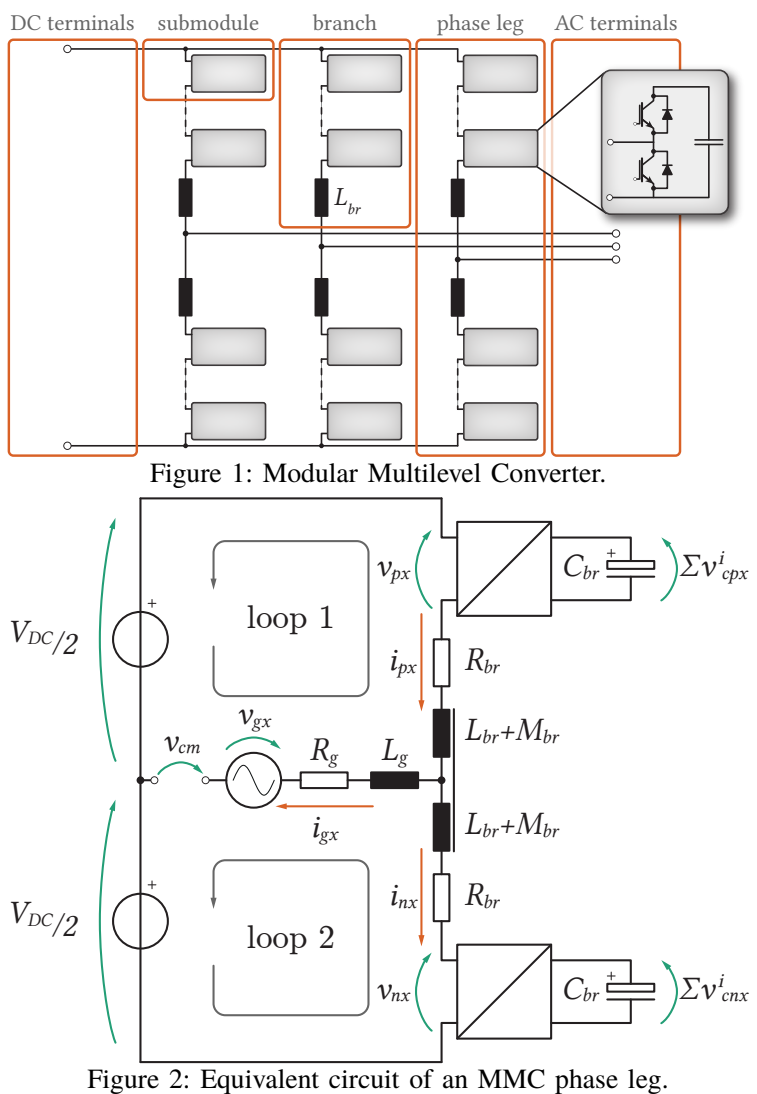

ternal quantities). Moreover, two branches, labeled as positive (upper) and negative (lower), comprise the phase leg. It is noteworthy that every SM hosts the capacitor $C_{\mathrm{SM}}$, which represents the floating energy source. As there is no external fixed voltage power supply to individual SMs, number of levels can be freely chosen and scaled to an application. Nevertheless, with the aim of maintaining the proper operation of the MMC, energy balancing among the SMs must be achieved. Balancing algorithm performance, in terms of control current capacity requirements and response time, can augment performance of the converter without any hardware alterations. Hence, comparing different balancing schemes during the converter design stage might be considered valuable.

Per-phase equivalent circuit of the MMC, derived from Fig. 1, is displayed in Fig. 2. Addition and subtraction of upper and lower Kirchhoff's Voltage Loop (KVL) equations reveals 
converter's controllability. From (1)-(2), it can be seen that grid current $i_{\mathrm{g}}$ and, MMC-specific, internal circulating current $i_{\mathrm{c}}$ are controllable through voltage components denoted by $v_{\mathrm{s}}$ and $v_{\mathrm{c}}$, respectively.

$$
\begin{aligned}
V_{\mathrm{dc}}-v_{\mathrm{c}} & =2\left(L_{\mathrm{br}}-M_{\mathrm{br}}\right) \frac{d i_{\mathrm{c}}}{d t}+2 R_{\mathrm{br}} i_{\mathrm{c}} \\
v_{\mathrm{s}}-v_{\mathrm{g}}-v_{\mathrm{CM}} & =\left(L_{\mathrm{g}}+\frac{L_{\mathrm{br}}+M_{\mathrm{br}}}{2}\right) \frac{d i_{\mathrm{g}}}{d t} \\
& +\left(R_{\mathrm{g}}+\frac{R_{\mathrm{br}}}{2}\right) i_{\mathrm{g}}
\end{aligned}
$$

In the above equations, the following notation is adopted: $v_{\mathrm{c}}=\left(v_{\mathrm{n}}+v_{\mathrm{p}}\right) / 2-$ total inserted leg voltage, $v_{\mathrm{s}}=\left(v_{\mathrm{n}}-v_{\mathrm{p}}\right) / 2$ - differential phase-leg voltage, $i_{\mathrm{c}}=\left(i_{\mathrm{p}}+i_{\mathrm{n}}\right) / 2$ - circulating current, $i_{\{\mathrm{p}, \mathrm{n}\}}=\left(i_{\mathrm{c}} \pm i_{\mathrm{g}}\right) / 2-$ positive and negative branch currents.

\section{BALANCING METHODS}

Energy balancing relies on the control of suitable circulating current components. It is of interest to control horizontal, vertical and total energy, i.e. distribution of charge among phase-legs, between upper and lower branch of each phase, and total charge of the MMC, respectively. Energy fluctuations are determined by branches' capacitors voltage dynamics as

$$
C_{\mathrm{br}} \frac{d v_{\mathrm{C} \Sigma\{\mathrm{p}, \mathrm{n}\}}}{d t}=m_{\{\mathrm{p}, \mathrm{n}\}}\left(\frac{i_{\mathrm{g}}}{2}+i_{\mathrm{c}}\right)
$$

where $C_{\mathrm{br}}, v_{\mathrm{C} \Sigma}$ and $m_{\{\mathrm{p}, \mathrm{n}\}}$ denote equivalent branch capacitance, sum capacitor voltage within a branch and the so-called insertion index, respectively. Assuming the closed-loop control of the MMC internal quantities [8], insertion indices can be obtained as

$$
m_{\{\mathrm{p}, \mathrm{n}\}}=\frac{v_{\mathrm{c}}^{*} \mp v_{\mathrm{s}}^{*}}{v_{\mathrm{C} \Sigma\{\mathrm{p}, \mathrm{n}\}}}
$$

Substitution of (4) into (3), while assuming $v_{\mathrm{c}}^{*} \approx V_{\mathrm{DC}} / 2$ and defining sum and differential phase-leg energies as $W_{\Sigma}=W_{\mathrm{p}}+W_{\mathrm{n}}$ and $W_{\Delta}=W_{\mathrm{p}}-W_{\mathrm{n}}$, revelas converter energy dynamics [9].

$$
\begin{aligned}
& \frac{d W_{\Sigma}}{d t}=\underbrace{V_{\mathrm{DC}} i_{\mathrm{c}}}_{\substack{P_{\mathrm{DC}} \text { and } \\
\text { balancing }}}-\underbrace{\frac{\hat{v}_{\mathrm{s}} \hat{i}_{\mathrm{g}}}{2} \cos (\varphi)}_{P_{\mathrm{AC}}}-\underbrace{\frac{\hat{v}_{\mathrm{s}} \hat{i}_{\mathrm{g}}}{2} \cos (2 \omega t+\varphi)}_{\text {zero average }} \\
& \frac{d W_{\Delta}}{d t}=\underbrace{\frac{V_{\mathrm{DC}} \hat{i}_{\mathrm{g}}}{2} \cos (\omega t+\varphi)}_{\text {zero average }}-\underbrace{2 i_{\mathrm{c}} \hat{v}_{\mathrm{s}} \cos (\omega t+\varphi)}_{\text {balancing }}
\end{aligned}
$$

A branch sum capacitor voltage contains the mean value along with the oscillating components being superimposed. Nevertheless, control of the $v_{\mathrm{C} \Sigma}$ mean value is normally ensured through the control actions, whereas proper selection of the SMs' capacitors limits its ripple [10]. From (5), the dual purpose of the circulating current DC component is revealed.
Firstly, it controls active power exchange with the DC link, equalizing it with the $\mathrm{AC}$ terminals power demand $\left(P_{\mathrm{AC}}\right)$. Secondly, it controls the energy exchange with the remaining converter legs, equalizing horizontal energy distribution. Further, based on (6), it is straightforward to conclude that the AC component of the circulating current $i_{\mathrm{c}}^{\sim}=\hat{i}_{\mathrm{c}}^{\sim} \cos (\omega t+\Psi)$ can be used to obtain the leg energy balance in vertical direction. Denoting the angle displacement between this component and the leg differential voltage component $v_{\mathrm{s}}$ by $\Phi$, yields

$$
\frac{d \overline{W_{\Delta}}}{d t}=-\hat{i}_{\mathrm{c}}^{\sim} \hat{v}_{\mathrm{s}} \cos (\Phi)
$$

Generally, $\Phi$ can be arbitrarily chosen, however, phase alignment of the above mentioned voltage and current components is assumed throughout this paper. In other words, $\Phi=0$.

Total energy control ensures matching of the powers at which the energy is exchanged between the DC and AC terminals through the adjustment of the DC link current $i_{\mathrm{DC}}^{*}$ Thus, circulating current can be expressed as

$$
i_{\mathrm{c}}=\underbrace{i_{\mathrm{DC}} / 3+i_{\mathrm{c} \Sigma}}_{=\overline{i_{\mathrm{c}}}}+\underbrace{i_{\mathrm{c} \Delta}}_{=i_{\mathrm{c}}}
$$

Nonetheless, horizontal (through $i_{\mathrm{c} \Sigma}$ ) and vertical (through $i_{\mathrm{c} \Delta}$ ) balancing actions are purely internal to the MMC and should not be seen either from the AC or the DC terminals. Consequently, the proper energy balancing criteria can be mathematically formulated as

$$
\sum \vec{i}_{\mathrm{c}\{\mathrm{a}, \mathrm{b}, \mathrm{c}\}}=i_{\mathrm{DC}}
$$

MMC energy balancing has been studied by many authors, providing multiple control strategies [11], [12]. This paper compares performance of two balancing methods proposed in [13], [14], in terms of required control current capacity and dynamics. According to the first method, horizontal balancing is achieved thorugh the adjustment of the circulating current components $i_{\mathrm{c} \Sigma}$ in the $\alpha \beta$ domain. Contrary to that, injection of reactive components into $i_{\mathrm{c} \Delta}$, as will be shortly explained, is used for the balancing in the vertical direction [13]. The second method relies on the leg-level energy controllers, followed by current reference mapping through an appropriate matrix [14].

\section{A. Method I}

Vertical energy balancing proposed in [13] essentially assumes two steps to satisfy the constraint defined by (9):

- Calculation of $i_{\mathrm{c} \Delta}$ circulating current amplitudes, inphase with $v_{\mathrm{s}}$ to achieve desired vertical energy corrections, as suggested by (7).

- For each injected active (in-phase) component, appropriate reactive (in-quadrature) components for remaining two phases are calculated to ensure that no disturbance caused by the control action is visible in DC link current. (9). Exemplary case presented in Fig. 3, provides detailed illustration of this step. It is noteworthy that reactive currents injected into a leg do not compromise the real power exchange with its environment. 

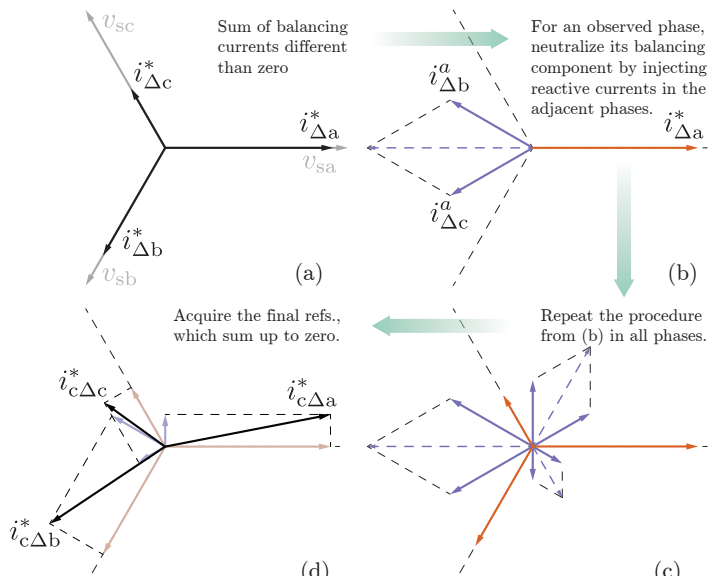

(c)

Figure 3: Principle of vertical energy balancing currents injection is shown from left to right. For control action in phase $a$, additional in-quadrature currents are injected into phases $b$ and $c$. The same is done for all three control action current references. This ensures no disturbance to be visible from the converter terminals.

From Fig. 3b, by observing phase $a$ balancing action $i_{\Delta \mathrm{a}}^{*}$, three current components can be derived as

$$
\begin{aligned}
& \underline{i_{\Delta \mathrm{a}}^{*}}=i_{\Delta \mathrm{a}}^{*} e^{j 0} \\
& \underline{i_{\Delta \mathrm{b}}^{a}}=\frac{i_{\Delta \mathrm{a}}^{*}}{2 \cos (\pi / 6)} e^{-j \frac{2 \pi}{3}} e^{-j \frac{\pi}{2}} \\
& \underline{i_{\Delta \mathrm{c}}^{a}}=\frac{i_{\Delta \mathrm{a}}^{*}}{2 \cos (\pi / 6)} e^{j \frac{2 \pi}{3}} e^{j \frac{\pi}{2}}
\end{aligned}
$$

Resulting balancing current references for all three phases can be converted to di 0 domain.

$$
\begin{aligned}
\underline{i}_{\Delta \mathrm{d}}^{*} & =\frac{1}{3}\left[\left|\underline{i}_{\Delta \mathrm{a}}^{*}\right|+\left|\underline{i}_{\Delta \mathrm{b}}^{*}\right|+\left|\underline{i}_{\Delta \mathrm{c}}^{*}\right|\right] \\
\underline{i}_{\Delta \mathrm{i}}^{*} & =\frac{2}{3}\left[\left|\underline{i}_{\Delta \mathrm{a}}^{*}\right|-\frac{1}{2}\left(\left|\underline{i}_{\Delta \mathrm{b}}^{*}\right|+\left|\underline{i}_{\Delta \mathrm{c}}^{*}\right|\right)\right] \\
& +j \frac{2}{3}\left[\frac{\sqrt{3}}{2}\left|\underline{i}_{\Delta \mathrm{b}}^{*}\right|-\frac{\sqrt{3}}{2}\left|\underline{i}_{\Delta \mathrm{c}}^{*}\right|\right] \\
\underline{i}_{\Delta 0}^{*} & =0
\end{aligned}
$$

Please notice that injection of reactive components discussed above ensures that balancing components of all legs sum up to zero, as confirmed by (13). If one recalls Clarke's transformation, an analogy can be made between the obtained di 0 components $\alpha \beta 0$ conversion matrix output.

$$
\underline{i}_{\Delta \mathrm{d}}^{*}=\left|i_{\Delta}^{*}\right|_{0} \quad \underline{i}_{\Delta \mathrm{i}}^{*}=\left|i_{\Delta}^{*}\right|_{\alpha}+j\left|i_{\Delta}^{*}\right|_{\beta}
$$

Implementation of vertical energy balancing is thus realized as presented in Fig. 4, where angle $\psi$ represents position of the $\overrightarrow{\boldsymbol{v}}_{\mathbf{s}\{\mathbf{a}, \mathbf{b}, \mathbf{c}\}}$ voltage vector in the stationary reference frame.

By analyzing sum phase-leg energies in $\alpha \beta 0$ domain, horizontal energy balancing can be conveniently realized.
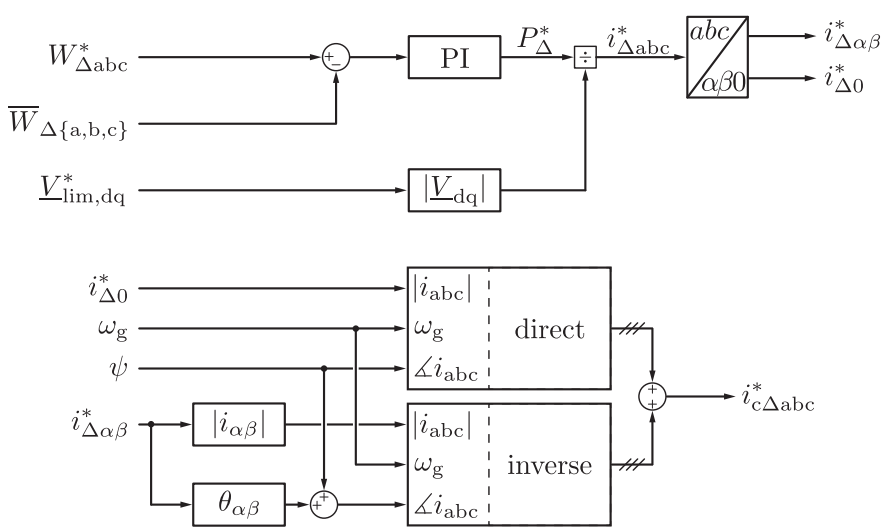

Figure 4: Implementation of vertical energy balancing control. Differential energy reference is naturally set as zero in $a b c$ system, to equalize vertical phase-leg-level energy distribution. Voltage amplitude is obtained directly from the Grid Current Control (GCC) $d q$ reference, as utilized Clarke's transformation is voltage amplitude invariant.

$$
\begin{aligned}
& W_{\Sigma \alpha}=\frac{2}{3}\left(W_{\Sigma \mathrm{a}}-\frac{W_{\Sigma \mathrm{b}}+W_{\Sigma \mathrm{c}}}{2}\right) \\
& W_{\Sigma \beta}=\frac{2}{3} \frac{\sqrt{3}}{2}\left(W_{\Sigma \mathrm{b}}-W_{\Sigma \mathrm{c}}\right) \\
& W_{\Sigma 0}=\frac{1}{3}\left(W_{\Sigma \mathrm{a}}+W_{\Sigma \mathrm{b}}+W_{\Sigma \mathrm{c}}\right)
\end{aligned}
$$

Setting $W_{\Sigma \alpha}$ and $W_{\Sigma \beta}$ to zero ensures that phase legs store the same amount of energy, determined by the common mode component $W_{\Sigma 0}$ (the image of the total MMC's stored energy).

$$
\begin{aligned}
& W_{\Sigma \beta}=0 \rightarrow W_{\Sigma \mathrm{b}}=W_{\Sigma \mathrm{c}} \\
& W_{\Sigma \alpha}=0 \rightarrow W_{\Sigma \mathrm{a}}=W_{\Sigma \mathrm{b}}=W_{\Sigma \mathrm{c}}
\end{aligned}
$$

Horizontal energy balancing action is implemented as depicted in Fig. 5. Filtered mean measured value of sum energies is fed to the controller with the aim driving $\alpha \beta$ components to zero.

Total energy control acts on zero-sequence circulating current component $i_{\mathrm{c} 0}=i_{\mathrm{DC}}^{*} / 3$, thus supplying energy from the $\mathrm{DC}$ link in order to satisfy the $\mathrm{AC}$ grid power requirements. Control action implementation is presented in Fig. 6.

Fig. 7 presents operation of balancing method discussed within this subsection. Simulation results were obtained in PLECS, using the MMC model specified in Table I. To test the converter performance, reference power profile presented in Fig. 8 was used. The leftmost zoom shows balanced

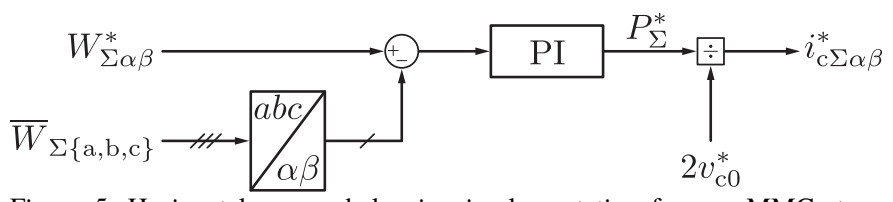

Figure 5: Horizontal energy balancing implementation for one MMC stage according to [13]. Observed energy is a filtered value, as average components are of interest. To achieve even horizontal energy distribution, $\alpha \beta$ components are referenced to zero.

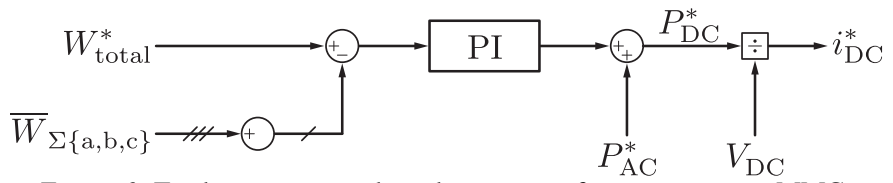

Figure 6: Total energy control implementation for inverter stage MMC. 


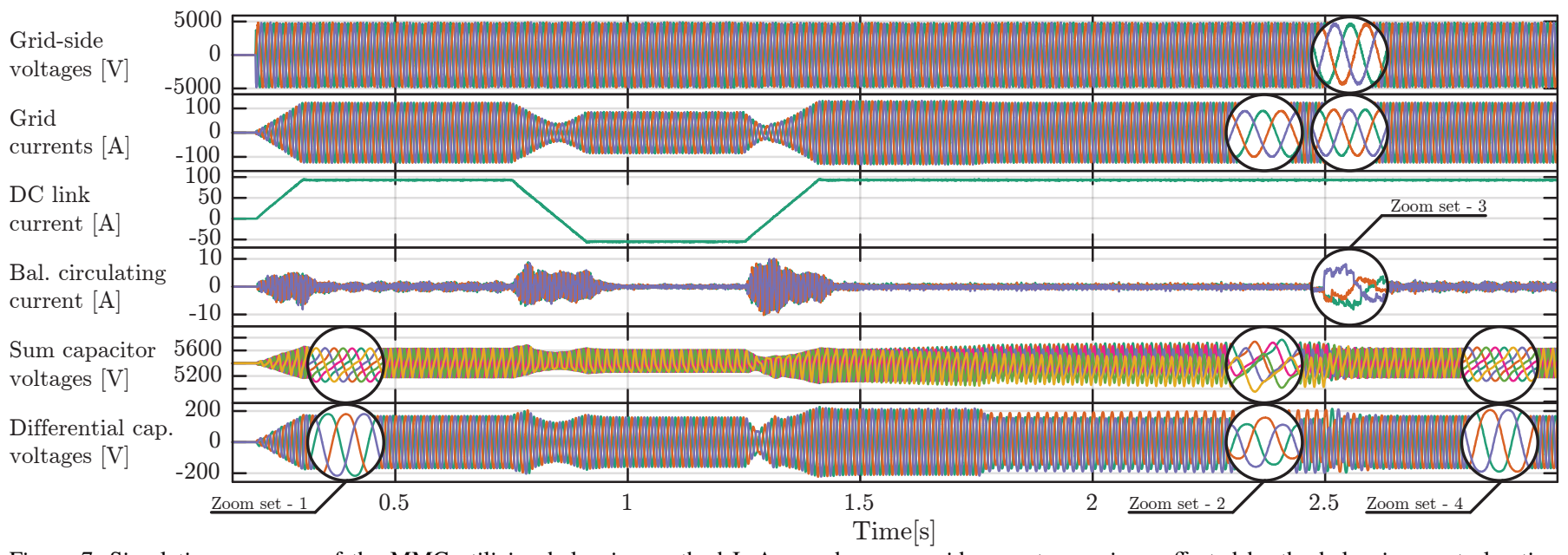

Figure 7: Simulation sequence of the MMC utilizing balancing method I. As can be seen, grid currents remain unaffected by the balancing control action at all times. During the period denoted by $T_{\text {dis }} \in[1.5 \mathrm{~s}, 2.5 \mathrm{~s})$, the energy balancing controller were disabled, which resulted in the SMs capacitor voltages divergence. Once the controllers were reactivated at time instant $t=2.5 \mathrm{~s}$, converter energies converged back to the nominal values.

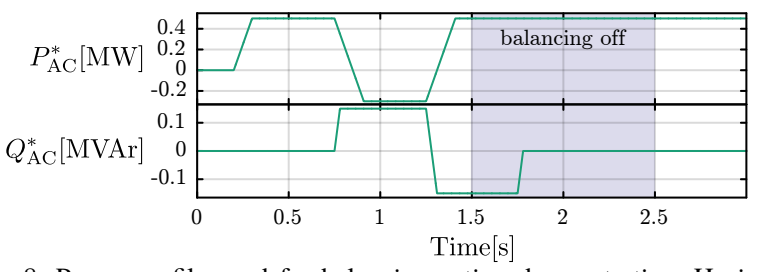

Figure 8: Power profile used for balancing action demonstration. Horizontal and vertical balancing are disabled from $1.5 \mathrm{~s}$ to $2.5 \mathrm{~s}$

sum and differential capacitor voltages. During the interval $T_{\text {dis }} \in[1.5 \mathrm{~s}, 2.5 \mathrm{~s})$, horizontal and vertical balancing actions were disabled. The second zoom set shows gradual divergence of the SMs' voltages despite the grid currents being unaltered, which is a consequence of the closed loop control. Upon reactivation of balancing controllers, circulating current action (third zoom set) reestablishes the energy balance leaving the grid currents unaffected.

\section{B. Method II}

Taking the independent balancing actions, in per-phase manner $\left(i_{\mathrm{c}, \text { leg }}^{*}\right)$, is not permitted in the MMC-alike structures, as constraint defined by (9) would be violated. A topologyindependent (universal) balancing approach is considered in [14], which assumes calculation of per-phase current references, followed by mapping through a suitable matrix which guarantees fulfillment of (9) at all times.

For now, direction of balancing will be neglected so that the results can be considered general. All the balancing actions

\begin{tabular}{lcc}
\multicolumn{3}{c}{ Table I: Test converter parameters. } \\
\hline \hline \multicolumn{1}{c}{ Parameter } & Label & Value \\
\hline Line voltage & $U_{\mathrm{n}}$ & $3.3 \mathrm{kV}$ \\
Apparent power & $S_{\mathrm{n}}$ & $0.5 \mathrm{MVA}$ \\
Grid frequency & $f_{\mathrm{n}}$ & $50 \mathrm{~Hz}$ \\
Number of SMs per branch & $N_{\mathrm{SM}}$ & 8 \\
SM voltage & $V_{\mathrm{SM}}$ & $675 \mathrm{~V}$ \\
SM capacitance (tolerance $\pm 5 \%)$ & $C_{\mathrm{SM}}$ & $2.25 \mathrm{mF}$ \\
Branch inductance & $L_{\mathrm{br}}$ & $2.5 \mathrm{mH}$ \\
Branch inductance coupling coefficient & $k_{\mathrm{br}}$ & 0.3 \\
Branch resistance & $R_{\mathrm{br}}$ & $20 \mathrm{~m} \Omega$ \\
Switching frequency & $f_{\mathrm{sw}}$ & $999 \mathrm{~Hz}$ \\
\hline \hline
\end{tabular}

being demanded by the energy controllers $\left(\Delta i_{\mathrm{c}, \mathrm{i}}\right)$ must sum up to zero in the DC link, which can be formulated as

$$
\underbrace{\left[\begin{array}{lll}
1 & 1 & 1
\end{array}\right]}_{T_{\mathrm{i}}} \underbrace{\left[\begin{array}{c}
\Delta i_{\mathrm{c}, \mathrm{a}} \\
\Delta i_{\mathrm{c}, \mathrm{b}} \\
\Delta i_{\mathrm{c}, \mathrm{c}}
\end{array}\right]}_{I}=0
$$

One can understand that matrix $T_{\mathrm{i}}$ represents the mapping $\mathbb{R}^{3} \rightarrow \mathbb{R}$ whereas all the vectors $I$ being mapped to zero do not actually corrupt the MMC DC link current. It is exactly this set of vectors that defines the null space (kernel) of matrix $T_{\mathrm{i}}$. Nonetheless, energy unbalance among the MMC branches/legs can take any arbitrary values resulting in the control actions violating the above constraint. To overcome this issue, a reference vector $I^{*}$, such that $T_{\mathrm{i}} \cdot I^{*} \neq 0$, can be projected to the null-space of $T_{\mathrm{i}}$, which allows for (20) to be satisfied. Fig. 9 provides an illustration of the above discussion.
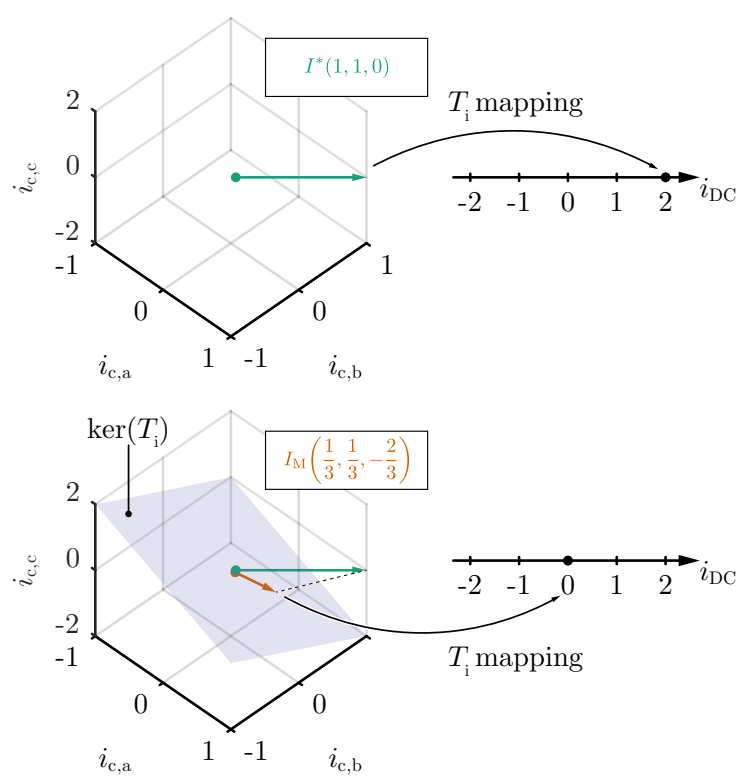

Figure 9: Projection of the reference vector $I^{*}$ which does not satisfy (20) to the null-space of transformation matrix $T_{\mathrm{i}}$ 
Basically, the problem of balancing comes down to identification of the suitable vector $I_{\mathrm{M}}$ which represents the projection of the original reference $I^{*}$ to the null-space of $T_{\mathrm{i}}$. For this purpose, the Singular Value Decomposition (SVD) can be used. If a random matrix $T_{\mathrm{i}}$ is applied to a set of basis vectors $\vec{V}_{\mathrm{i}}$, the latter are stretched and rotated, consequently providing a set of new basis vectors $\vec{u}_{i}$.

$$
T_{\mathrm{i}}^{\left[\begin{array}{lll}
\vec{V}_{1} & \ldots & \vec{V}_{\mathrm{n}}
\end{array}\right]}=\underbrace{\left[\begin{array}{lll}
\vec{u}_{1} & \ldots & \vec{u}_{\mathrm{n}}
\end{array}\right]}_{U}[\underbrace{\left[\begin{array}{ccc}
\sigma_{1} & \ldots & 0 \\
\vdots & \sigma_{\mathrm{r}} & 0 \\
0 & \ldots & 0
\end{array}\right]}_{\Sigma}
$$

The above equation can be rearranged as

$$
T_{\mathrm{i}}=U \Sigma V^{*}
$$

In (22), $V^{*}$ represents conjugate transpose of $V$. If $V$ is real, $V^{*}=V^{\mathrm{T}}$ is true, and (22) can be simplified.

$$
\underbrace{T_{\mathrm{i}}}_{\mathrm{mxn}}=\underbrace{U}_{\mathrm{mx} \text { m m x n n n }} \underbrace{\Sigma} \underbrace{V^{\mathrm{T}}}
$$

It is noteworthy that projection of the reference vector on the null-space of $T_{\mathrm{i}}$ provides the solution satisfying the least squares criteria. In other words, the head of every vector different from $I_{\mathrm{M}}$, and being in $\operatorname{ker}\left(T_{\mathrm{i}}\right)$, falls further from the head of $I^{*}$ when compared to $I_{\mathrm{M}}$ itself. However, null-space of a matrix needs to be firstly defined and SVD also provides the means for its simple identification. Since U and V from (22) are unitary (rotation) matrices, it is clear that neither of them can compress the space (re-scale its grid). Therefore,

$$
\operatorname{ker}\left(T_{\mathrm{i}}\right)=\operatorname{ker}\left(U \Sigma V^{\mathrm{T}}\right)=\operatorname{ker}\left(\Sigma V^{\mathrm{T}}\right)
$$

Also, (23) can be expressed as

$$
T_{\mathrm{i}}=\underbrace{\left[\begin{array}{ll}
\vec{U}_{\mathrm{R}} & \vec{U}_{\mathrm{N}}
\end{array}\right]}_{\mathrm{m} \times \mathrm{m}} \underbrace{\left[\begin{array}{cc}
S_{\mathrm{rxr}} & 0 \\
0 & 0
\end{array}\right]}_{\mathrm{m} \times \mathrm{n}} \underbrace{\left[\begin{array}{c}
\vec{V}_{\mathrm{R}}^{*} \\
\vec{V}_{\mathrm{N}}^{*}
\end{array}\right]}_{\mathrm{n} \times \mathrm{n}}
$$

It can be seen from the above equation that matrix $\Sigma$ contains a square submatrix with elements different than zero and $n-r$ zero vectors. Number of zero vectors in $\Sigma$ corresponds to the number of orthogonal vectors comprising a basis for $\operatorname{ker}\left(T_{\mathrm{i}}\right)$ (a set of vectors $B=\left\{\vec{V}_{1}, \ldots, \vec{V}_{n}\right\}$ is said to be the basis of a space $S$ if all the vectors from $B$ are linearly independent while every vector from $S$ can be expressed through their linear combination). To prove this statement, one can initially start with the proof of (24). If (24) holds, there must be a vector $\vec{\omega}$ such that $\Sigma V^{\mathrm{T}} \vec{\omega}=0$, which implies that $U \Sigma V^{\mathrm{T}} \vec{\omega}=0 \Rightarrow \operatorname{ker}\left(\Sigma V^{\mathrm{T}}\right) \subset \operatorname{ker}\left(U \Sigma V^{\mathrm{T}}\right)$. The opposite should also be proven, namely $\operatorname{ker}\left(U \Sigma V^{\mathrm{T}}\right) \subset \operatorname{ker}\left(\Sigma V^{\mathrm{T}}\right)$. Assuming that this is not true leads to $\Sigma V^{\mathrm{T}} \vec{\omega} \neq 0$ and $U \Sigma V^{\mathrm{T}} \vec{\omega}=0$. Since $\mathrm{U}$ is unitary matrix $(\operatorname{det}(U)=1)$ it is clear that two previously presented equations can not be true unless $\Sigma V^{\mathrm{T}} \vec{\omega}=0$.
To find a basis of $\operatorname{ker}\left(\Sigma V^{\mathrm{T}}\right), \Sigma$ shall be observed for the moment. All the vectors $\vec{e}_{\mathrm{k}}$, for which $\sigma_{k}=0$, can be considered as the orthonormal basis of $\operatorname{ker}(\Sigma)$. Once all the vectors $\vec{e}_{k}$ are identified, one can say that vectors $V_{\vec{e}_{i}}$ represent the basis of $\operatorname{ker}\left(\Sigma V^{\mathrm{T}}\right)$. This can be shown as

$$
\Sigma V^{\mathrm{T}} V_{\vec{e}_{i}}=\Sigma \underbrace{V^{\mathrm{T}} V}_{\text {Identity matrix }} \quad \vec{e}_{i}=\Sigma \vec{e}_{i}=0
$$

Application of matrix $V$ to the vectors $\vec{e}_{\mathrm{k}}$ results in isolation of the $k$-th column from the matrix $V$. Moreover, since $\vec{e}_{\mathrm{k}}$ are orthogonal, so are $V_{\vec{e}_{\mathrm{k}}}$.

Once the orthogonal basis of $\operatorname{ker}\left(\Sigma V^{\mathrm{T}}\right)$ is identified, vector demanded by the converter balancing controllers $I^{*}$ must be mapped into a suitable vector $I_{\mathrm{M}}$. If basis vectors $B=\left\{\vec{V}_{\mathrm{e}_{1}}, \ldots, \vec{V} \mathrm{e}_{k}\right\}$ are at disposal, the following relation follows.

$$
B^{\mathrm{T}} I^{*}=\left[\begin{array}{c}
\vec{V}_{\mathrm{e}_{1}} \\
\vec{V}_{\mathrm{e}_{2}} \\
\vdots \\
\vec{V}_{\mathrm{e}_{k}}
\end{array}\right] I^{*}=\underbrace{\left[\begin{array}{c}
<\vec{V}_{\mathrm{e}_{1}} \cdot I^{*}> \\
<\vec{V}_{\mathrm{e}_{2}} \cdot I^{*}> \\
\vdots \\
<\vec{V}_{\mathrm{e}_{k}} \cdot I^{*}>
\end{array}\right]}_{\text {scalar products }}
$$

Vector on the right hand side of (27) contains scalar products of orthogonal basis vectors and a balancing reference vector $I^{*}$. These scalar products actually represent projections of a vector $I^{*}$ onto the basis vectors $\vec{V}_{\mathrm{e}_{\mathrm{i}}}$. However, these are the coordinates of a vector $I^{*}$ in a new coordinate system consisting of vectors $\vec{V}_{\mathrm{e}_{\mathrm{i}}}$. To obtain the coordinates of a new vector in the conventional reference frame, vector $B^{\mathrm{T}} I^{*}$ needs to be multiplied by $B$, which yields

$$
\begin{aligned}
B \cdot B^{\mathrm{T}} \cdot I^{*} & =\left[\begin{array}{llll}
\vec{V}_{\mathrm{e}_{1}} & \vec{V}_{\mathrm{e}_{2}} & \ldots & \vec{V} \mathrm{e}_{k}
\end{array}\right]\left[\begin{array}{c}
<\vec{V}_{\mathrm{e}_{1}} \cdot I^{*}> \\
<\vec{V}_{\mathrm{e}_{2}} \cdot I^{*}> \\
\vdots \\
<\vec{V}_{\mathrm{e}_{k}} \cdot I^{*}>
\end{array}\right] \\
& =\sum_{k} \vec{V}_{\mathrm{e}_{k}}\left\langle\vec{V}_{\mathrm{e}_{k}} \cdot I^{*}\right\rangle
\end{aligned}
$$

An advantage of the presented principle is that once the control current components are identified per leg, one should only define converter terminal constraints to apply the matrix presented in (28).

Null-space of the matrix $T_{\mathrm{i}}$ from (20) can be identified by firstly creating its SVD form, from which matrix $V$ can be easily identified.

$$
T_{\mathrm{i}}=\underbrace{\left[\begin{array}{lll}
-1
\end{array}\right]}_{U} \underbrace{\left[\begin{array}{lll}
\sqrt{3} & 0 & 0
\end{array}\right]}_{\Sigma} \underbrace{\left[\begin{array}{ccc}
-\frac{\sqrt{3}}{3} & -\frac{\sqrt{3}}{3} & -\frac{\sqrt{3}}{3} \\
-\frac{\sqrt{3}}{3} & \frac{3+\sqrt{3}}{6} & -\frac{1}{3+\sqrt{3}} \\
-\frac{\sqrt{3}}{3} & -\frac{1}{3+\sqrt{3}} & \frac{3+\sqrt{3}}{6}
\end{array}\right]}_{V^{\mathrm{T}}}
$$




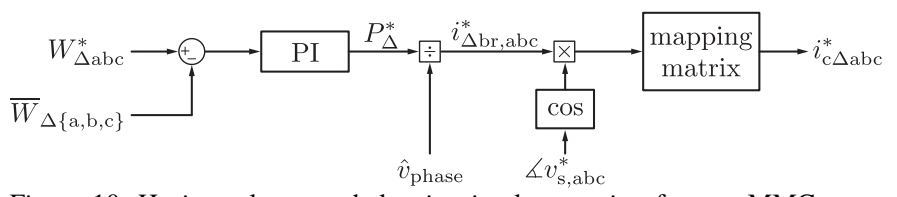

Figure 10: Horizontal energy balancing implementation for one MMC stage according to [14]. Observed energy is a filtered value, as average components are of interest. Per-phase reference values are driven to zero.

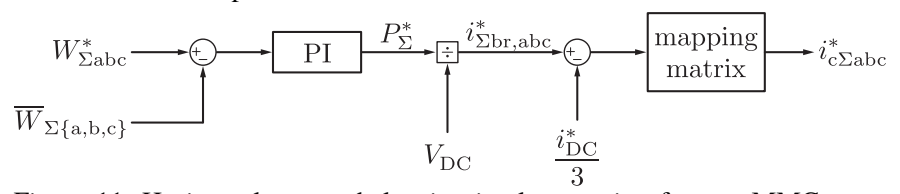

Figure 11: Horizontal energy balancing implementation for one MMC stage according to [13]. Observed energy is a filtered value, as average components are of interest. Per-phase references are set to one third of total energy reference.

According to the previously presented principles, orthonormal basis of $T_{\mathrm{i}}$ consists of two vectors being in the second and third column of $V$ (second and third element in $\Sigma$ are zero).

$$
B=\left[\begin{array}{cc}
-\frac{\sqrt{3}}{3} & -\frac{\sqrt{3}}{3} \\
\frac{3+\sqrt{3}}{6} & -\frac{1}{3+\sqrt{3}} \\
-\frac{1}{3+\sqrt{3}} & \frac{3+\sqrt{3}}{6}
\end{array}\right]
$$

Following (28), matrix that maps vector of references $I^{*}$ into a vector of suitable balancing components $I_{\mathrm{M}}$ equals

$$
B B^{\mathrm{T}}=\frac{1}{3}\left[\begin{array}{ccc}
2 & -1 & -1 \\
-1 & 2 & -1 \\
-1 & -1 & 2
\end{array}\right]
$$

Current references for vertical, horizontal and total energy control are calculated per-phase, and corrected through the mapping matrix defined by (31). Total energy controller is not affected by different balancing approach, as it operates on the converter level. Vertical and horizontal energy balancing controllers are realized as depicted in Figs. 10 and 11. As converter terminal constraints are the same for the either of balancing directions, matrix from (31) is used in both cases.
Sequence of operation of the balancing method presented is this subsection can be seen in Fig. 12. As in Fig. 7, converter of specifications given in Table I is loaded with the reference power profile from Fig. 8. Zoomed areas show that circulating currents' balancing action is decoupled from grid conditions. After reestablishment of balancing control capability, capacitors' voltages converge back to reference values.

\section{Comparison}

\section{A. Mapping of references}

Resulting from very different ways of reference mapping, the highest difference was expected in vertical energy imbalance suppression. Prior to running the simulations, corrective actions of the two methods were investigated, for three different cases of vertical imbalance. Exemplary current vectors are presented in Fig. 13.

In case where reference currents are already balanced (the energy mismatch in vertical direction is the same in all legs), no alteration is performed, as expected. For non-identical amplitudes of the same sign, one can notice the two methods output similar corrections, with the second one providing slightly lower circulating current amplitudes. In cases where original references are imbalanced and of different signs, however, difference between balancing methods is significant and evidently on the side of the second method in terms of amplitudes. Notwithstanding, a major difference between the presented methods refers to the preservation of power components being in charge of balancing. Namely, injection of reactive currents with the aim of balancing the converter in vertical direction (method I), does not alter the active powers being demanded by the balancing controllers. However, alteration of circulating currents caused by the adequate matrix mapping (method II) causes the balancing power components to differ from the reference ones. Consequently, method I can be considered power invariant, however, at the expense of demanding higher circulating currents compared to method II, under the same operating scenario.

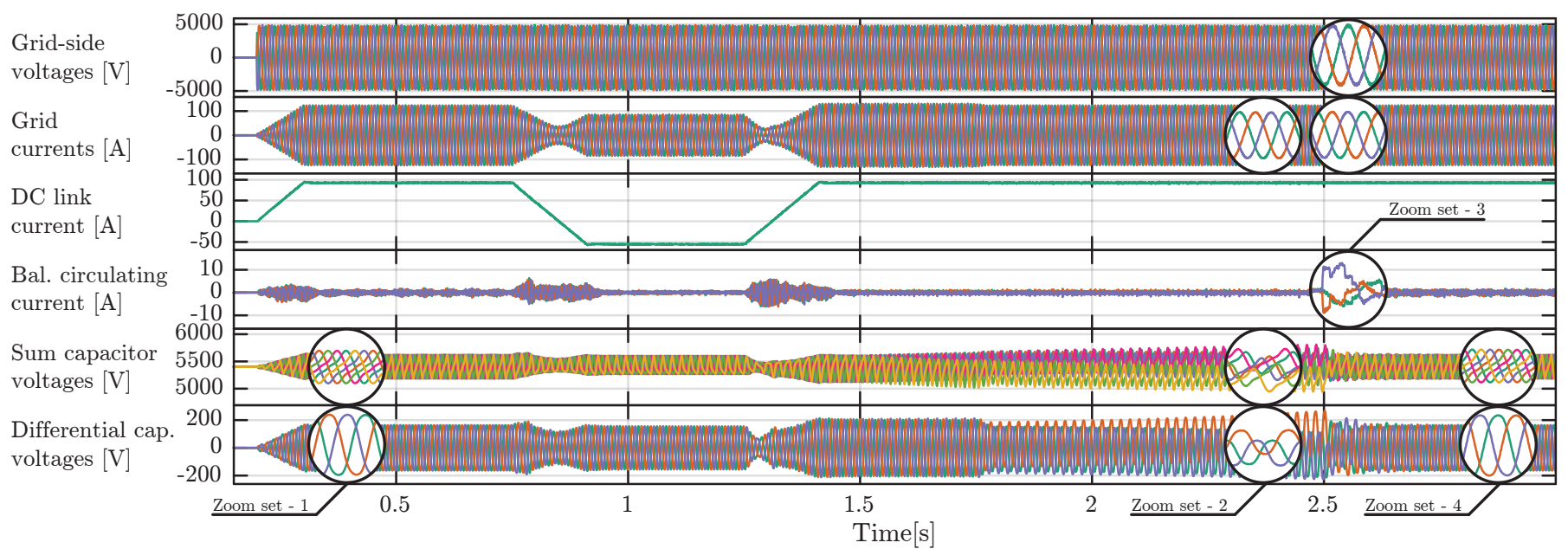

Figure 12: Simulation sequence of the MMC utilizing balancing method II. As can be seen, grid currents remain unaffected by the balancing control action at all times. During the period denoted by $T_{\text {dis }} \in[1.5 \mathrm{~s}, 2.5 \mathrm{~s})$, the energy balancing controller were disabled, which resulted in the SMs capacitor voltages divergence. Once the controllers were reactivated at time instant $2.5 \mathrm{~s}$, converter energies converged back to the nominal values. 

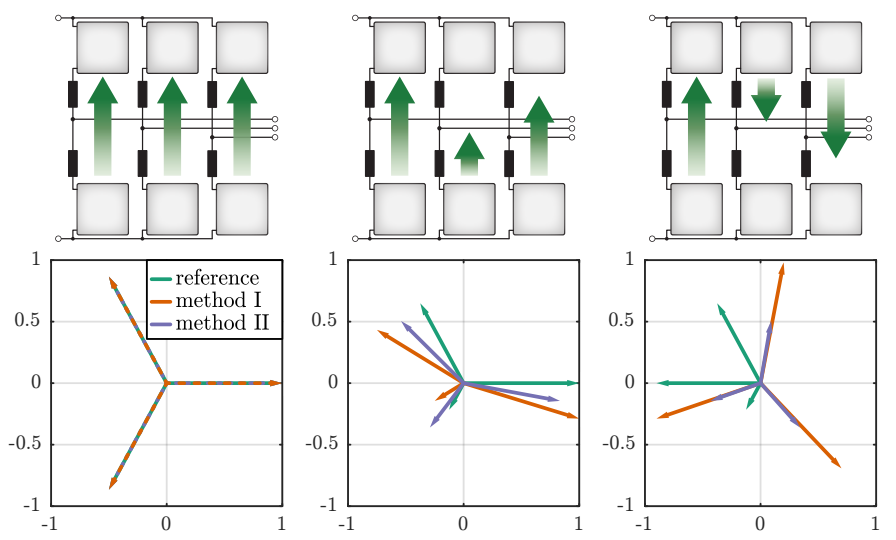

Figure 13: Reference mapping ensured by two compared vertical balancing methods. Original references, reactive current injection (method I) and corrected per-phase mapping (method II) results are seen in green, orange and violet, respectively. In the first case, references are already of the same sign and magnitude, and there is no corrective action as they sum up to zero. The third case, where balancing references are of different signs, shows significant difference in terms of amplitudes calculated from two analyzed approaches.

\section{B. Test scenarios}

Comparison of the two balancing methods has been performed for multiple scenarios, to evaluate performance differences. MMC was operated as inverter, assuming stable DC link voltage, at power of exchange towards the grid of

$$
P_{\mathrm{AC}}=400 \mathrm{~kW} \quad Q_{\mathrm{AC}}=100 \mathrm{kVAr}
$$

Looking at Fig. 13, three cases are of interest for vertical energy balancing:

- Equal imbalance in all phase-legs.

- Unequal imbalance in phase-legs, all deviations being of the same sign.

- Unequal imbalance in phase-legs, deviations being of arbitrary signs.
Table II: MMC energy balancing controllers' sampling times.

\begin{tabular}{lcc}
\hline \multicolumn{1}{c}{ Loop } & Label & Value \\
\hline Grid current control & $T_{\mathrm{gcc}}$ & $1 / f_{\mathrm{sw}}$ \\
Circulating current control & $T_{\mathrm{ccc}}$ & $T_{\mathrm{sw}}$ \\
Vertical energy balancing & $T_{\Delta}$ & $25 T_{\mathrm{sw}}$ \\
Horizontal energy balancing & $T_{\Sigma}$ & $15 T_{\mathrm{sw}}$ \\
Total energy balancing & $T_{\mathrm{tot}}$ & $15 T_{\mathrm{sw}}$ \\
\hline \hline
\end{tabular}

Concerning horizontal energy balancing, two cases are investigated:

- Equal imbalance between one phase-leg and the remaining two.

- Unequal imbalance between one phase-leg and the remaining two.

Ultimately, to obtain fair results, energy controllers were tuned identically for both methods, as presented in Table II.

\section{Simulation Results}

Following the adopted test scenarios, simulations were performed using PLECS. Results for vertical and horizontal energy balancing are presented in Fig. 14. Uppermost schematics depict reference imbalance scenarios $\left(W_{\Delta}^{*}\right.$ - leg energy mismatches in vertical direction, $\Delta W_{\Sigma}^{*}$ - leg energy mismatches in horizontal direction), whereas the middle graphs represent the responses of converter energies to the previously defined step reference changes. For the sake of comparison, responses for both balancing methods were presented on the single graph. It is noteworthy that $W_{\Delta}^{*}$ and $\Delta W_{\Sigma}^{*}$ are normally set as zero, however to test the controllers performance, these references were set to values different than zero. From the dynamics point of view, this case does not differ the one implying zero mismatch references with initial mismatch conditions differing it. Lowermost graph displays circulating currents for the two balancing methods. Please note that, for easier comparison
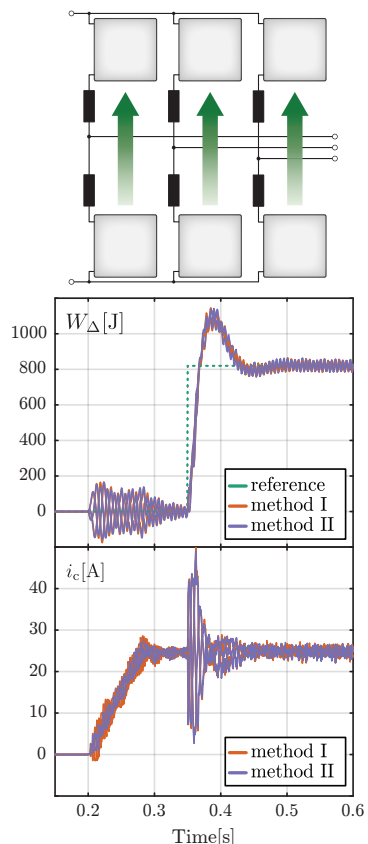
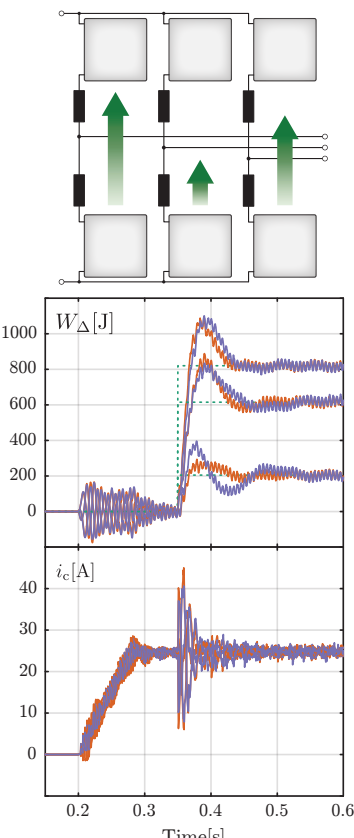
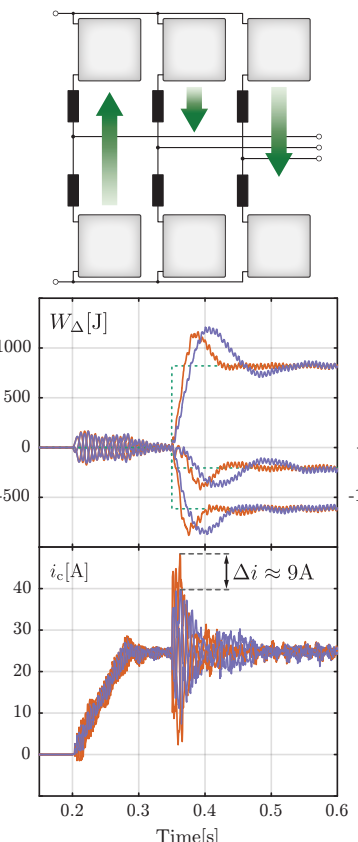
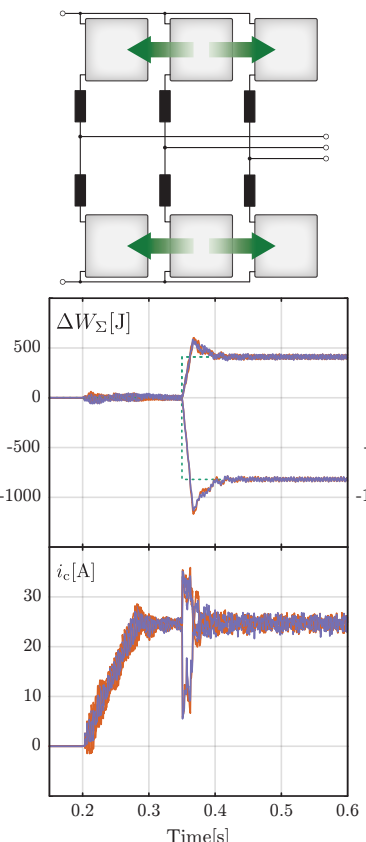
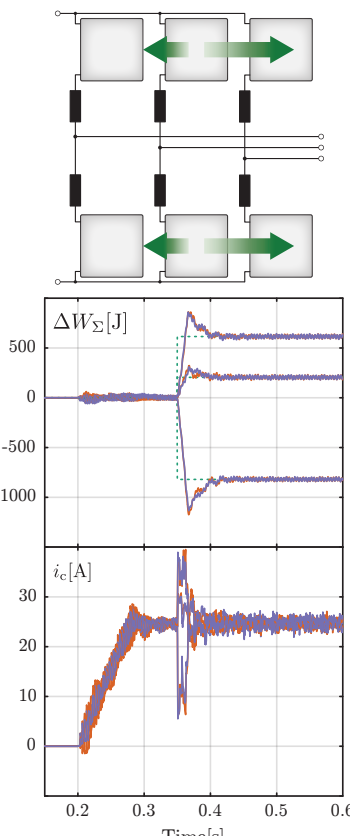

Figure 14: Comparison of the two balancing methods has been performed for multiple scenarios of vertical and horizontal energy imbalance, to evaluate response differences. Three phases of a balancing approach are of the same color, for easier comparison of the methods. 
of the methods, all three phases of a particular balancing approach are of the same color.

Horizontal balancing actions were performed almost identically for the two approaches. Both settling time and required circulating current amplitude are very similar. Type of imbalance hasn't played a role in results.

Regarding vertical balancing actions under equal imbalances in all phases, expectedly, no difference was observed among the two approaches. In cases where vertical imbalance rate differs among phase-legs, it can be noticed that settling time is shorter utilizing [13], at the expense of higher balancing current amplitude. In this particular case, for the same imbalance scenario and converter parameters, amplitude of circulating currents is $10-20 \%$ lower for [14].

\section{Conclusion}

This paper presented comparison of the two MMC energy balancing methods [13], [14]. Control strategies of both methods have been thoroughly presented, and operation verified on an arbitrary load cycle. A series of PLECS simulations has been performed to cover typical energy imbalance cases. Vertical balancing method using reactive current injection approach [13] performed faster in terms of settling times, while corrected per-phase control [14] required lower control current capacity for the control purposes. The first balancing method ensures preservation of the original power balancing references generated by energy controllers. On the other hand, the second considered method aims at finding the output current values nearest to the original references, without violating converter terminal constraints, not guaranteeing exact active power values for the balancing action. Resulting corrected current references can therefore be lower in amplitude compared to the first method, while settling time is longer. This result is visible in normal operation under the same conditions. Comparing Fig. 7 and Fig. 12, for load profile given in Fig. 8, balancing components of circulating current (8) are of higher amplitude for method I, as expected.

\section{ACKNOWLEDGMENT}

This work has been supported by Swiss Federal Office of Energy (SFOE), Swiss Inovation Agency (CTI) and Swiss Competence Center for Energy Research (SCCER FURIES).

\section{REFERENCES}

[1] P. K. Steimer, O. Senturk, S. Aubert, and S. Linder, "Converter-fed synchronous machine for pumped hydro storage plants," 2014 IEEE Energy Conversion Congress and Exposition, ECCE 2014, pp. 4561-4567, 2014.

[2] A. Lesnicar and R. Marquardt, "An innovative modular multilevel converter topology suitable for a wide power range," 2003 IEEE Bologna PowerTech - Conference Proceedings, vol. 3, pp. 272-277, 2003.
[3] K. Friedrich, "Modern hvde plus application of vsc in modular multilevel converter topology," in 2010 IEEE International Symposium on Industrial Electronics, Jul. 2010, pp. 3807-3810.

[4] M. Pereira, D. Retzmann, J. Lottes, M. Wiesinger, and G. Wong, "Svc plus: An mmc statcom for network and grid access applications," in 2011 IEEE Trondheim PowerTech, IEEE, 2011, pp. 1-5.

[5] D. Weiss, M. Vasiladiotis, C. Banceanu, N. Drack, B. Odegard, and A. Grondona, "Igct based modular multilevel converter for an ac-ac rail power supply," in PCIM Europe 2017; International Exhibition and Conference for Power Electronics, Intelligent Motion, Renewable Energy and Energy Management, May 2017, pp. 1-8.

[6] M. Vasiladiotis, R. Baumann, H. Christoph, and J. Steinke, "IGCT-based Direct AC / AC Modular Multilevel Converters for Pumped Hydro Storage Plants," pp. 4837-4844, 2018.

[7] M. Basić, P. C. O. Silva, and D. Dujić, "High Power Electronics Innovation Perspectives for Pumped Storage Power Plants," 2018.

[8] A. Antonopoulos, L. Angquist, and H. Nee, "On dynamics and voltage control of the modular multilevel converter," in 2009 13th European Conference on Power Electronics and Applications, Sep. 2009, pp. 1-10.

[9] K. Sharifabadi, L. Harnefors, H.-P. Nee, S. Norrga, and R. Teodorescu, Design, control, and application of modular multilevel converters for HVDC transmission systems. John Wiley \& Sons, 2016.

[10] K. Ilves, S. Norrga, L. Harnefors, and H. Nee, "On energy storage requirements in modular multilevel converters," IEEE Transactions on Power Electronics, vol. 29, no. 1, pp. 77-88, Jan. 2014.

[11] S. Wang, G. P. Adam, A. M. Massoud, D. Holliday, and B. W. Williams, "Analysis and Assessment of Modular Multilevel Converter Internal Control Schemes," IEEE Journal of Emerging and Selected Topics in Power Electronics, vol. PP, no. c, pp. 1-1, 2019.

[12] A. Christe and D. Dujic, "Modular Multilevel Converter Control Methods Performance Benchmark for Medium Voltage Applications," IEEE Transactions on Power Electronics, vol. 34, no. 5, pp. 4967-4980, 2019.

[13] P. Munch, D. Gorges, M. Izak, and S. Liu, "Integrated current control, energy control and energy balancing of Modular Multilevel Converters," IECON 2010 36th Annual Conference on IEEE Industrial Electronics Society, pp. 150-155, 2010.

[14] A. J. Korn, M. Winkelnkemper, P. Steimer, and J. W. Kolar, "Capacitor voltage balancing in Modular Multilevel Converters," 